ARTICLE

Received 23 Sep 2015 | Accepted 11 Apr 2016 | Published 18 May $2016 \quad$ DOl: 10.1038/ncomms11590 OPEN

\title{
Structural basis for haem piracy from host haemopexin by Haemophilus influenzae
}

\author{
Silvia Zambolin ${ }^{1}$, Bernard Clantin², Mohamed Chami ${ }^{3}$, Sylviane Hoos ${ }^{4}$, Ahmed Haouz ${ }^{5}$, Vincent Villeret ${ }^{2}$
} \& Philippe Delepelaire ${ }^{1}$

Haemophilus influenzae is an obligate human commensal/pathogen that requires haem for survival and can acquire it from several host haemoproteins, including haemopexin. The haem transport system from haem-haemopexin consists of $\mathrm{HxuC}$, a haem receptor, and the two-partner-secretion system $\mathrm{HxuB} / \mathrm{HxuA}$. HxuA, which is exposed at the cell surface, is strictly required for haem acquisition from haemopexin. HxuA forms complexes with haem-haemopexin, leading to haem release and its capture by HxuC. The key question is how HxuA liberates haem from haemopexin. Here, we solve crystal structures of HxuA alone, and $\mathrm{HxuA}$ in complex with the $\mathrm{N}$-terminal domain of haemopexin. A rational basis for the release of haem from haem-haemopexin is derived from both in vivo and in vitro studies. HxuA acts as a wedge that destabilizes the two-domains structure of haemopexin with a mobile loop on HxuA that favours haem ejection by redirecting key residues in the haem-binding pocket of haemopexin.

\footnotetext{
${ }^{1}$ CNRS, Université Paris 7 UMR 7099, Institut de Biologie Physico-Chimique, 13 rue Pierre et Marie Curie, 75005 Paris, France. ${ }^{2}$ Université de Lille, CNRS, UMR 8576 - UGSF, Unité de Glycobiologie Structurale et Fonctionnelle, Laboratoire de Biologie Structurale Intégrative, F 59000 Lille, France. ${ }^{3}$ Center for Cellular Imaging and Nano Analytics (C-CINA), Biozentrum, University of Basel, Mattenstrasse 26, CH-4058 Basel, Switzerland. ${ }^{4}$ Institut Pasteur, Plate-forme de Biophysique, CNRS-UMR 3528, 25, rue du Dr Roux, 75724 Paris Cedex 15, France. ${ }^{5}$ Institut Pasteur, Plate-forme de Cristallographie, CNRS-UMR 3528 , 25 , rue du Dr Roux, 75724 Paris Cedex 15, France. Correspondence and requests for materials should be addressed to P.D. (email: philippe.delepelaire@ibpc.fr).
} 
aem-binding proteins, which contain $>75 \%$ of the iron in higher organisms, are the major sources of iron for all commensal/pathogenic organisms that develop in mammals. Haem uptake from host haemoproteins requires a recognition step, and an internalization step. Internalization across the cell membranes is usually the only step that requires energy. Energy is provided by proton-motive-force dissipation (TonB-dependent receptors) to cross the outer membrane of Gram-negative bacteria ${ }^{1}$ and ATP hydrolysis (in most cases, via ATPase subunits associated with cytoplasmic membrane bound permeases) to cross the cytoplasmic membrane of both Gram-positive and Gram-negative bacteria ${ }^{2,3}$. Haem can then be used as such or degraded via various pathways to retrieve the iron atom.

Haemophilus influenzae is a Gram-negative bacterium uniquely found in humans that asymptomatically colonizes the nasopharynx of up to $40 \%$ of the population. On crossing the epithelium barrier, it can cause several diseases, from otitis to pneumoniae and meningitis, mostly in young children and immunocompromised patients. Besides its iron requirement, $H$. influenzae also displays an absolute haem prerequisite to sustain its aerobic growth, as it is devoid of most haem biosynthesis enzymes ${ }^{4}$. For this reason it has developed several systems that allow the acquisition of haem from host haemoproteins, such as haemoglobin, haemoglobin-haptoglobin and haemopexin ${ }^{5,6}$. The haem-haemopexin acquisition system is particularly interesting because Haemophilus influenzae is one of the few bacterial species that can use this haemoprotein as a source of haem and iron.

Haemopexin is a $50-\mathrm{kDa}$ serum glycoprotein involved in haem recycling after haemolysis. It has one of the highest known affinities for haem ( $\mathrm{Kd}$ ca. $0.1 \mathrm{pM}$ ), and is present in all mammalian body fluids including cerebrospinal fluid ${ }^{7}$. Its concentration (in normal conditions $\sim 10 \mu \mathrm{M}$ in serum) increases on inflammation. The X-ray structure of rabbit haem-haemopexin has been solved (PDB codes 1QHU and 1QJS). It is made up of two homologous halves (four-bladed $\beta$-propeller architecture, defining the matrix metalloproteinase MMP fold) connected by a ca. 20 residues linker. Haem is located at the boundary of the two MMP domains, two histidine residues (one from the linker, one from the C-terminal domain) acting as iron axial ligands ${ }^{8}$.

The system that allows haem uptake from haemopexin (Hxu system), comprises three proteins: $\mathrm{HxuB}$ and HxuA, which together form a two-partner-secretion (Tps) system $^{9-11}$, and the TonB-dependent haem receptor HxuC. In invasive $H$. influenzae strains, HxuA is exposed at the cell surface. It belongs to the family of TpsA cargo proteins that are secreted through their specific TpsB partner. TpsB's are members of a superfamily of 16 -stranded $\beta$-barrel proteins involved in protein secretion across, and protein incorporation into, the outer membrane. TpsA proteins are cell surface/secreted virulence factors, and can be divided into at least two subfamilies: the filamentous haemagglutinin adhesin (FHA) subfamily, and the highmolecular-weight adhesin (HMW) subfamily (to which HxuA belongs). The HMW subfamily is defined by the presence of a disulfide bridge at the C-terminus, involved in anchoring the TpsA at the cell surface. In both subfamilies the highly conserved $\mathrm{N}$-terminal Tps domain is involved in the recognition/secretion by the TpsB partner ${ }^{11}$. Previous studies showed that HxuA makes high-affinity stoichiometric complexes with haemopexin or haem-haemopexin and that haem is released from haem-haemopexin on interaction with HxuA, becoming available for the receptor HxuC (ref. 12). To our knowledge, no full-length structure of a TpsA protein is available. However, in silico models predict the fold of these proteins as long $\beta$-helices ${ }^{11}$.
The only structures reported so far are of Tps domains from several TpsA proteins ${ }^{13-16}$.

There are few systems where capture of haem from host haemoproteins by the bacterium is understood at the molecular level, and the Hxu system appears to be well-suited to tackle the open questions. Here we report the crystal structures of full-length HxuA and HxuA in complex with the N-terminal domain of rabbit haemopexin, together with HxuA mutants characterization, both in vivo and in vitro. We propose a detailed molecular mechanism for haem acquisition from haemopexin.

\section{Results}

Crystal structure of HxuA. HxuA (884 residues) contains two cysteine residues close to the C-terminal end, at positions 855 and 861. These two residues are thought to form a disulfide bridge, which likely anchors the protein in its TpsB translocator ${ }^{17}$. A variant of HxuA was engineered, in which these two cysteines were replaced by serines, allowing more efficient release of the protein into the supernatant ${ }^{12}$. The $\mathrm{X}$-ray structure of this variant (referred to as HxuA throughout) was solved at $1.6 \AA$ resolution, and is presented in Fig. 1a together with the crystallographic data (Table 1, Supplementary Fig. 1a). HxuA is an elongated molecule that folds into a ca. $120 \times 35 \times 35 \AA$ kinked right-handed $\beta$-helix; it is made up of two distinct parts of roughly equal length. The $\mathrm{N}$-terminal domain (residues 1-350, encompassing the TPS domain ${ }^{14}$ ) is characteristic of this family of proteins, and referred to as the secretion domain. The C-terminal domain (residues 351-884) is by anticipation referred to as the functional domain. C-terminal residues $818-884$ were not seen in the electron density map. However, mass spectrometry analysis of a dissolved crystal indicated that the protein was largely intact in the crystal (Supplementary Fig. 2a, bottom, as compared with Supplementary Fig. 2a, top). The crystal structure of the Tps secretion domain of HxuA has been reported previously ${ }^{14}$. Except for crystal contacts, there do not appear to be significant differences, between this domain in its isolated form and in the full-length protein.

The resolution of the structure of full-length HxuA reveals that the protein is fully organized as a right-handed $\beta$-helix, with three parallel $\beta$-sheets PB1, PB2 and PB3 (Supplementary Table 1 for the definition of secondary structures). PB2 and PB3 each have 24 strands and are continuous, while PB1 is split into two parts, one part containing 11 strands (towards the $\mathrm{N}$-terminus) and the other 13 strands (towards the C-terminus). These two parts are separated by a 10 residue-long $\alpha$-helix (H1) and a 21 residue-long insertion between strands $\beta 36$ and $\beta 39$. This insertion contains two short $\beta$-strands $\beta 37 / \beta 38$ ( $\beta 37$ (Tyr314-Asn316)/ $\beta 38$ (Arg320Asp322), Supplementary Table 1) that lie nearly perpendicular to the axis of the C-terminal part of the $\beta$-helix. At the junction between the secretion and the functional domains, strands $\beta 40$ and $\beta 41$ are also swapped within PB2 (Fig. 1b). Together these features induce a kink in the relative orientations of the secretion and functional domains and a twist in the $\beta$-helix itself, which might ensure a strong physical link between the two parts as well as some plasticity at the junction of the two domains. The C-terminal functional domain contains very long insertions between the strands of the parallel $\beta$-sheets. These insertions cover one face of the $\beta$-helix formed by PB3. All of the insertions or loops are thus folded onto the floor of a single parallel $\beta$-sheet, giving the $\mathrm{C}$-terminal region of the molecule a highly asymmetric shape. The longest insertion (residues 512-578), located between strands $\beta 57$ (in PB3) and $\beta 60$ (in PB2) is folded onto most of the C-terminus, and contains additional secondary structure including helices H3, H4 and H5 (Fig. 1a,c). This loop also 
a

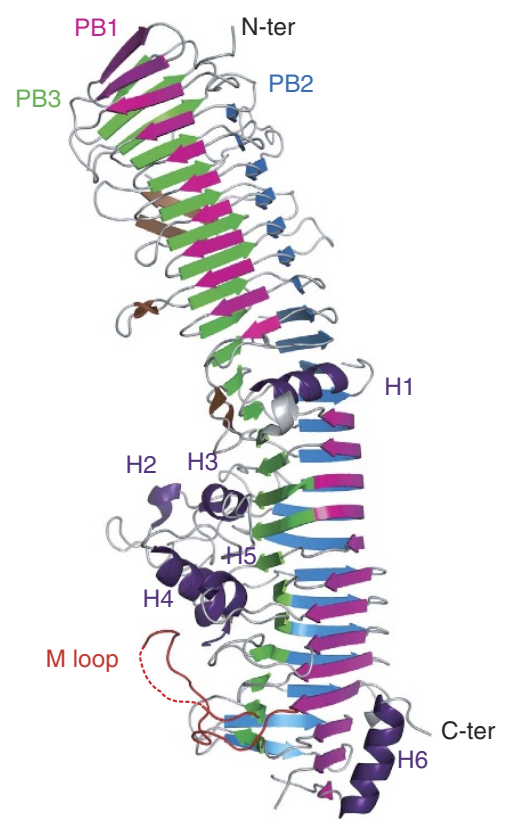

b

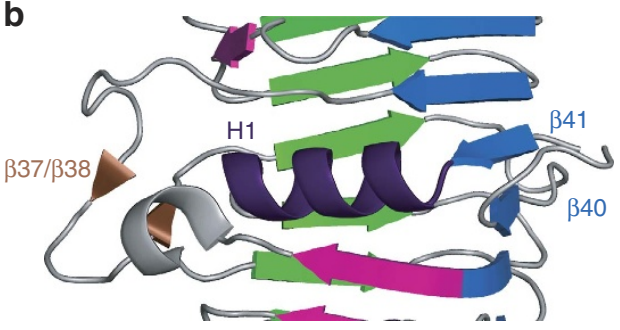

C

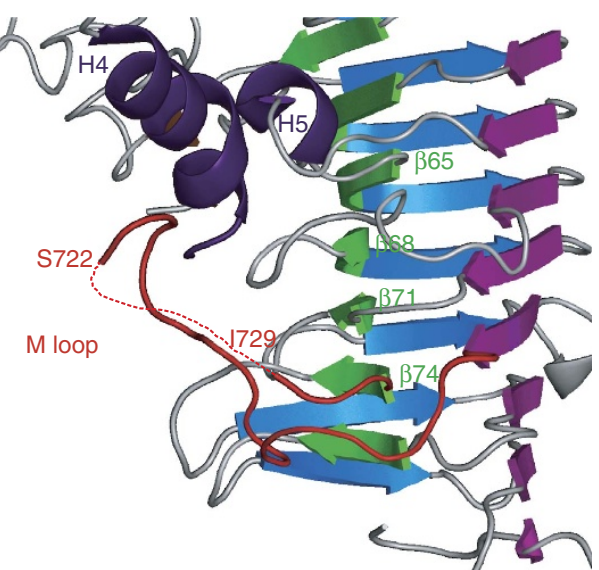

Figure 1 | X-ray structure of the HxuA molecule. (a) Cartoon representation of HxuA, a right-handed $\beta$-helix. Parallel $\beta$-sheet PB1 is coloured in magenta, PB2 in blue, PB3 in green and the extra-helix strand motifs in brown. The $\alpha$-helix elements $\mathrm{H} 1-\mathrm{H} 6$ are shown in purple. (b) Enlarged view of $\alpha$-helix $\mathrm{H} 1$, which splits PB1 into two parts. Together with the extra motifs $\beta 37 / \beta 38$ and the swap of $\beta 40$ and $\beta 41$, this element induces a twist in the $\beta$-helix, highlighting the separation between the secretion domain and the functional domain. (c) Enlarged view of loop 706-731, in red, referred to as the $\mathrm{M}$ loop, which undergoes an important conformational change during complex formation.

\begin{tabular}{|c|c|}
\hline & HxuA \\
\hline \multicolumn{2}{|l|}{ Data collection } \\
\hline Space group & $\mathrm{P} 2{ }_{1} 2_{1} 2$ \\
\hline \multicolumn{2}{|l|}{ Cell dimensions } \\
\hline$a, b, c(\AA)$ & $93.40,177.13,54.94$ \\
\hline$\alpha, \beta, \gamma\left(^{\circ}\right)$ & $90.00,90.00,90.00$ \\
\hline Resolution $(\AA)$ & $1.60(1.65-1.60)$ \\
\hline$R_{\text {merge }}$ & $6.6(124.4)$ \\
\hline$|/ \sigma|$ & $12.71(1.74)$ \\
\hline Completeness (\%) & $98.8(98.0)$ \\
\hline Redundancy & $5.6(5.5)$ \\
\hline \multicolumn{2}{|l|}{ Refinement } \\
\hline Resolution $(\AA)$ & 1.60 \\
\hline No. reflections & 119,491 \\
\hline$R_{\text {work }} / R_{\text {free }}$ & $0.188 / 0.211$ \\
\hline \multicolumn{2}{|l|}{ No. atoms } \\
\hline Protein & 6,026 \\
\hline Ligand/ion & / \\
\hline Water & 737 \\
\hline \multicolumn{2}{|l|}{ B-factors } \\
\hline Protein & 34.25 \\
\hline Ligand/ion & - \\
\hline Water & 46.78 \\
\hline \multicolumn{2}{|l|}{ R.m.s. deviations } \\
\hline Bond lengths $(\AA)$ & 0.010 \\
\hline Bond angles $\left({ }^{\circ}\right)$ & 1.16 \\
\hline
\end{tabular}

Values in parentheses are for the highest resolution shell.

contains two short additional distorted $\beta$-strands $(\beta 58 / \beta 59)$. Several segments within loops are not seen in the electron density map and, given the overall resolution of the structure, likely correspond to mobile portions of the molecule. One loop, hereafter referred to as the $M$ (for moving) loop (residues 706-731, between strands $\beta 73$ and $\beta 74$ in, respectively, PB1 and PB3), which we showed to play an essential role in haem release from haemopexin (see below) is not completely defined in the electron density map (residues 723-728 are missing). The $\mathrm{M}$ loop conformation is stabilized by several $\mathrm{H}$-bonds/salt bridges (Supplementary Table 2) involving residues from the 713-722 segment locking the loop in a groove delineated by strands $\beta 65, \beta 68, \beta 71, \beta 74$, helices $\mathrm{H} 4$ and $\mathrm{H} 5$ and the $\beta 64-\beta 65$ loop (Fig. 1c). Finally, a C-terminal 12 residue-long amphipathic $\alpha$-helix (H6, residues $802-814$ ) is folded back onto PB1 and faces strands $\beta 73, \beta 76, \beta 79$ and $\beta 80$, with its polar residues oriented towards the exterior of the molecule. Hydrophobic interactions with strands from PB1 stabilize its conformation.

HxuA only interacts with the N-terminal domain of haemopexin. The resolution of the HxuA structure led us to propose that the interaction surface of HxuA with haemopexin is probably provided by the long insertions and structural motifs folded on PB3 of the C-terminal part of the $\beta$-helix. However, it gave no clues about how this could happen and how haem is released during the interaction of HxuA with haem-haemopexin. Haemopexin is made of two structurally similar, four-bladed $\beta$-propeller MMP domains (residues 1-208 and 228-435) connected by a flexible interdomain linker that contains one of the two haem iron ligands (histidine 213 in the case of rabbit haemopexin), the other one (His 266) being in the C-terminal domain $^{8}$. It was reported previously that the two propeller domains can be separated by mild proteolytic digestion ${ }^{18}$. The isolated N-terminal domain (residues 1-208) still has some affinity for haem, and both domains have affinity for each other 
that is reinforced in the presence of haem ${ }^{18}$. It was, therefore, relevant to investigate whether HxuA had affinity for both haemopexin propeller domains or just one. To this end, we purified both domains separately, and tested their putative association with $\mathrm{HxuA}$ by isothermal titration calorimetry (ITC) and size exclusion chromatography (SEC).

We previously demonstrated that HxuA forms high-affinity stoichiometric complexes with haemopexin $(\Delta \mathrm{H}=-47.3$ $\mathrm{kcal} \mathrm{mol}^{-1}, \mathrm{Ka}>10^{9} \mathrm{M}^{-1}$ ) (ref. 12). Figure 2 shows the thermograms of the interaction of HxuA with isolated domains of haemopexin, and with full-length haemopexin as a control. No heat exchange signal was detected with the C-terminal haemopexin domain (CtHpx), but a very strong signal was observed with the $\mathrm{N}$-terminal haemopexin domain ( $\mathrm{NtHpx}$ ). Data analysis showed that a ca. 1:1 complex was formed in the latter case, and that the heat evolved during the interaction was almost equal to that observed with the full-length protein ( -48.5 versus $-52.7 \mathrm{kcal} \mathrm{mol}^{-1}$ in this particular experiment). This indicates that NtHpx likely provides most, if not all, of the bonds responsible for the interaction with $\mathrm{HxuA}$, with little or no contribution from the C-terminal domain. The affinity constant measured for NtHpx is smaller than that determined for the fulllength protein (Supplementary Table 3). The results were confirmed by SEC; NtHpx formed complexes with HxuA but CtHpx did not (see Supplementary Fig. 2b,d,e).

Transmission Electron Microscopy Negative Stain. We used negative stain transmission electron microscopy (TEM) to obtain additional topological information about the interactions between HxuA and haemopexin. In agreement with the X-ray data, HxuA was shown to have a ca. $140 \AA$ long and, on average, a $50 \AA$ wide rod-like structure (Supplementary Fig. 3a). At this resolution, the asymmetric shape of one end attributable to the C-terminal $\beta$-helix insertions, is not obvious. By contrast, for haemopexin two domains corresponding to the two MMP domains were clearly identified (Supplementary Fig. 3b).
Binding of NtHpx to HxuA resulted in extra density at one end of the elongated HxuA molecule, while the other end appeared more regular, and might thus be the $\mathrm{N}$-terminal part of the $\beta$-helix (Fig. 3a). The complex formed by HxuA with full-length haemopexin (HxuA-Hpx), had even more density at the same HxuA end (Fig. 3b). We attribute this additional density to the C-terminal domain of Hpx. Notably, the extra density does not always occupy the same position with respect to the rest of the molecule, but is found in a limited number of preferential positions, as documented by the class averages (Fig. 3c). These results suggest that, most likely due to the flexible linker between the two MMP domains, the C-terminal domain of Hpx does not occupy a fixed position in the HxuA-Hpx complex.

$\mathrm{X}$-ray structure of the HxuA-NtHpx complex. All attempts to obtain crystals of full-length haemopexin in complex with HxuA failed, probably because the linker joining the two domains is flexible and the C-terminal domain occupies several positions. This, together with the demonstrated interaction of HxuA with the N-terminal domain of haemopexin (Section 2) led us to undertake the crystallization of the HxuA-NtHpx complex. This complex gave many hits on high-throughput crystallization screening, and its structure was solved at $2.8 \AA$ resolution by molecular replacement with both our previous HxuA structure and the $\mathrm{N}$-terminal domain of haem-haemopexin derived from the 1QHU structure ${ }^{8}$ as templates (Fig. $4 \mathrm{a}$, Supplementary Fig. 1b and Table 2). NtHpx binds at the C-terminal end of HxuA. The overall shape of the HxuA molecule is well conserved between the free and bound states (rmsd $=0.76 \AA$ over $757 \mathrm{C} \alpha$ atoms (773 total), excluding residues $712-723$ from the $M$ loop, Ser765-Lys766 from another C-terminal loop making contacts with haemopexin, and residues Asn221 and Ala223 from another loop in the $\mathrm{N}$-terminal part), as is the shape of the $\mathrm{N}$-terminal domain of haemopexin ( $\mathrm{rmsd}=0.58 \AA$ over all $\mathrm{C} \alpha$ atoms). The N-terminal domain of haemopexin folds as a four-bladed $\beta$-propeller, with each propeller blade comprised of a three- or four-stranded, antiparallel $\beta$-sheet, the first and fourth blades
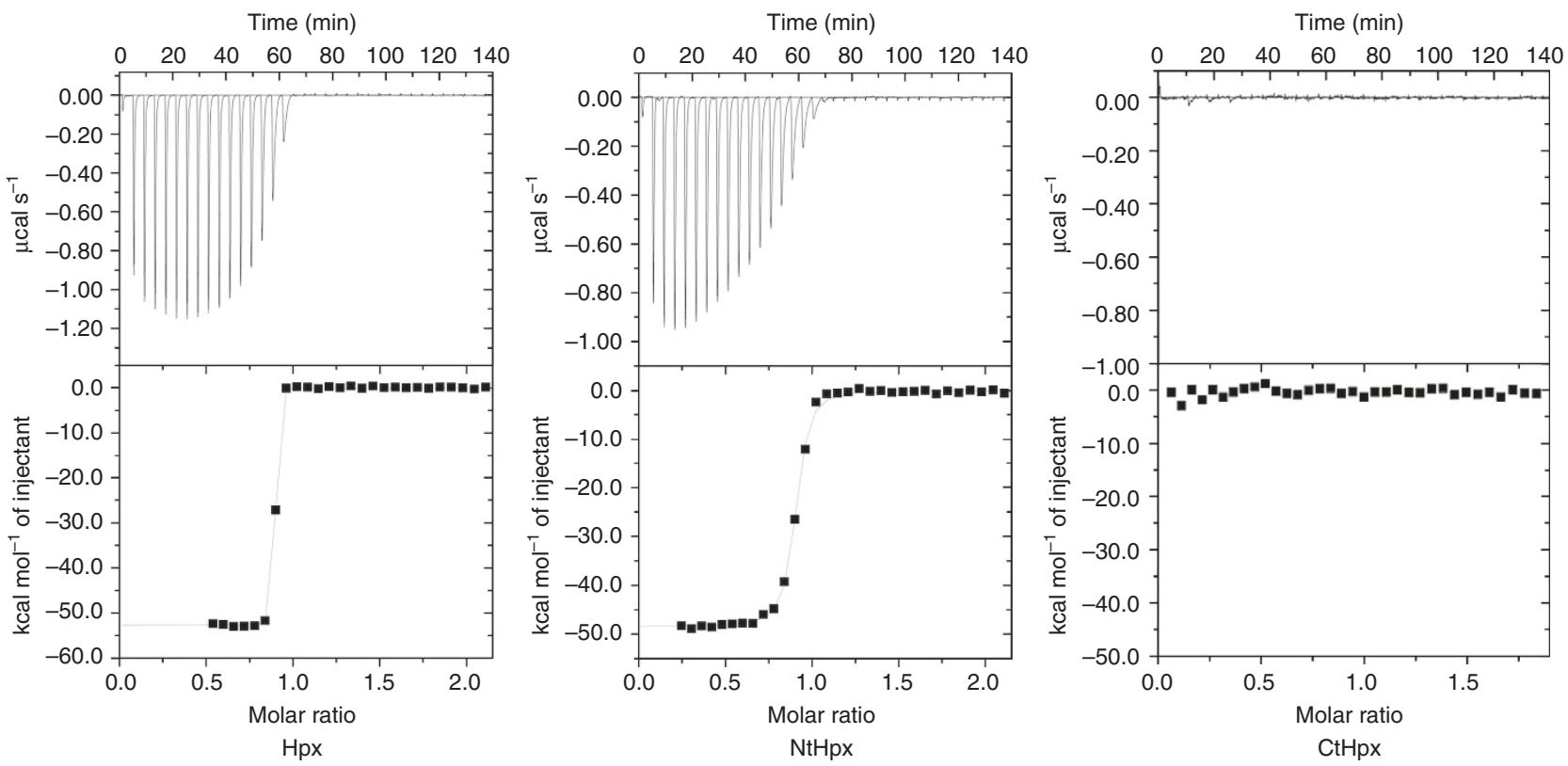

Figure 2 | Interaction of HxuA with haemopexin and its domains. Interaction of HxuA with haemopexin (Hpx) and the $\mathrm{N}$ and $\mathrm{C}$-terminal fragments of haemopexin ( $\mathrm{NtHpx}$ and $\mathrm{CtHpx}$, respectively), was measured by ITC. The upper part shows the heat signal for the titration. The lower part of each panel shows the binding isotherm derived from the heat signal, together with the fit calculated with Origin software. 

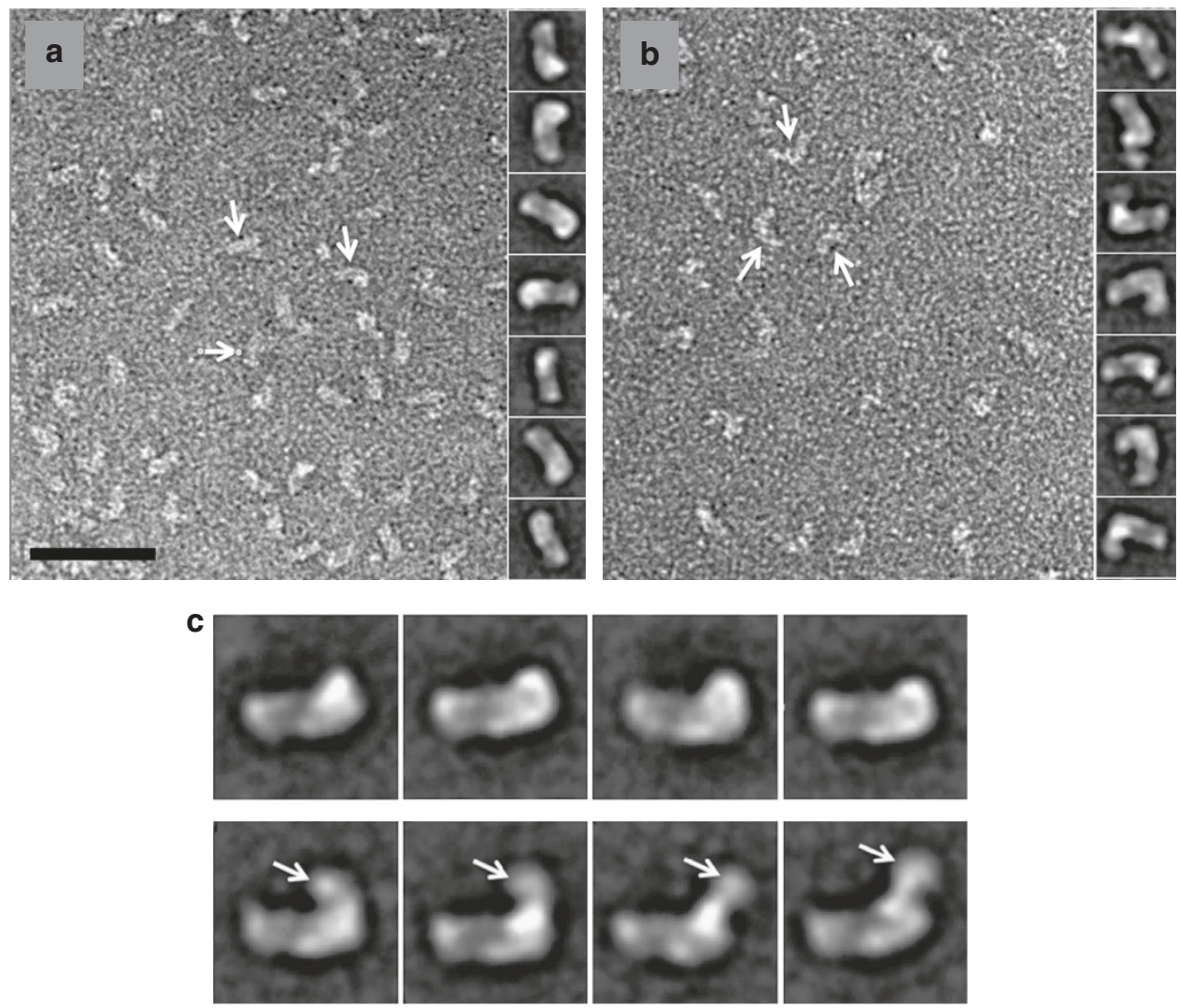

Figure 3 | TEM analysis of the HxuA-NtHpx and HxuA-Hpx complexes. (a) Micrograph of the negatively stained HxuA-NtHpx complex. The arrows indicate the protein particles adsorbed on the carbon film. The right panel shows a representative gallery of class averages from 7,104 particle projections analysed using the EMAN2 software. (b) Micrograph of the negatively stained HxuA-Hpx complex. The arrows indicate the protein particles adsorbed on the carbon film. The right panel shows a representative gallery of class averages from 9,847 particle projections analysed using the EMAN2 software. In c, the arrow indicates the extra density clearly visible on the side views of the HxuA-Hpx complex (bottom) compared with the HxuA-NtHpx complex (top panel). The scale bar in $\mathbf{a}$ is $50 \mathrm{~nm}$. The boxes are $18 \mathrm{~nm}$ in $\mathbf{a}$ and $\mathbf{b}$ and $20 \mathrm{~nm}$ in $\mathbf{c}$

being tied together by a disulphide bond between Cys27 and Cys208 (ref. 8). The respective orientation of the two molecules in the complex is such that the fourfold symmetry axis of the $\mathrm{NtHpx}$ four-bladed $\beta$-propeller is almost parallel to the axis of the HxuA $\beta$-helix (angle of ca. $20^{\circ}$ ). The surface of interaction (Fig. 4b) buries $\sim 1600 \AA^{2}$ and the contact zone is highly polar (Supplementary Table 4 and Fig. 4c). The region between residues 706 and 731, which we named the M (moving) loop, is of particular importance. This loop undergoes a very large movement (26 $\mathrm{A}$ for example for $\mathrm{C} \alpha$ of Gly718) on haemopexin binding. In HxuA alone, the $\mathrm{M}$ loop is locked in a groove delineated by strands $\beta 65, \beta 68, \beta 71, \beta 74$, helices $\mathrm{H} 4$ and $\mathrm{H} 5$, and the $\beta 64-\beta 65$ loop. The folding of two short $\beta$-strands between residues Glu713-Ile716 ( $\left.\beta 73^{\prime}\right)$ and Ala719-Ser722 ( $\beta 73$ '; see, for example, Fig. $4 \mathrm{~d}-\mathrm{f}$ ) of the $\mathrm{M}$ loop is induced by haemopexin binding, and occurs concomitant to the large conformational change. These $\beta$-strands contribute many interactions with blade 4 of haemopexin (Supplementary Table 4). In haemopexin, the interaction with HxuA only involves residues from blades 3 and 4 (Fig. 4a). First, within blade 3, residues located between strands 1 and 2 , between strands 3 and 4 , and within strand 4 interact with helix $\mathrm{H} 4$ and loops $\beta 51-\beta 52, \beta 57-\beta 60, \beta 67-\beta 68, \beta 71-72$ and $\beta 74-$ $\beta 75$. Second, within blade 4 , residues located in strands 2 and 3 and also in the insertion between strands 3 and 4 interact with residues from strands $\beta 71, \beta 73$ ', $\beta 73$ " and loops between strands $\beta 73$ " $-\beta 74$ and $\beta 79-\beta 80$. All polar interactions are listed in Supplementary Table 4 . Thus, the surface of interaction can roughly be divided into two patches, one coming from the moving $\mathrm{M}$ loop, and the other from regions of the $\beta$-helix that undergo much less extensive rearrangement on haemopexin binding and are set free on M loop movement (Fig. 4c).

Close examination of the structure of the HxuA-NtHpx complex also provides a rational basis for haem release on interaction of haem-haemopexin with HxuA. Superposition of the complete haem-haemopexin structure on the $\mathrm{NtHpx}$ in the complex indicates that the C-terminal domain of haemopexin would sterically clash with HxuA, mostly with loops $\beta 57-\beta 60$, $\beta 64-\beta 65$ and $\beta 67-\beta 68$ of HxuA that are folded over the $\beta$-helix, as well as with the M loop (Fig. $4 \mathrm{~d}$ ). Furthermore, analysis of the $M$ loop contacts with $\mathrm{NtHpx}$ also indicates that there would be a direct steric clash between Val-Ile714-715, Gly-Ala-Pro718-720 and both haem propionates in the haem-binding site in haemopexin (Fig. 4d). Indeed, residues of the $M$ loop make polar bonds with residues from haemopexin involved in haem binding and distort its binding pocket. In particular, in haemhaemopexin, residues Arg174 and 185 (blade 4; strands 2 and 3 respectively) make polar bonds with one of the propionates of the haem molecule. In the complex, their side chain orientation is modified, further weakening haem binding to $\mathrm{Hpx}$ (Fig. 4e,f). Moreover Arg185 from haemopexin makes polar bonds with side chains of residues Asp726 (loop $\beta 73$ "- $\beta 74$ ) and Glu713 ( $\beta 73$ "), while Hpx Arg174 is further stabilized by interactions with the carbonyl groups of Ile716 and Ala719 within HxuA (Supplementary Table 4). We therefore hypothesized that the whole-M-loop and in particular some of its residues would play an essential role in haem ejection from haem-haemopexin. 

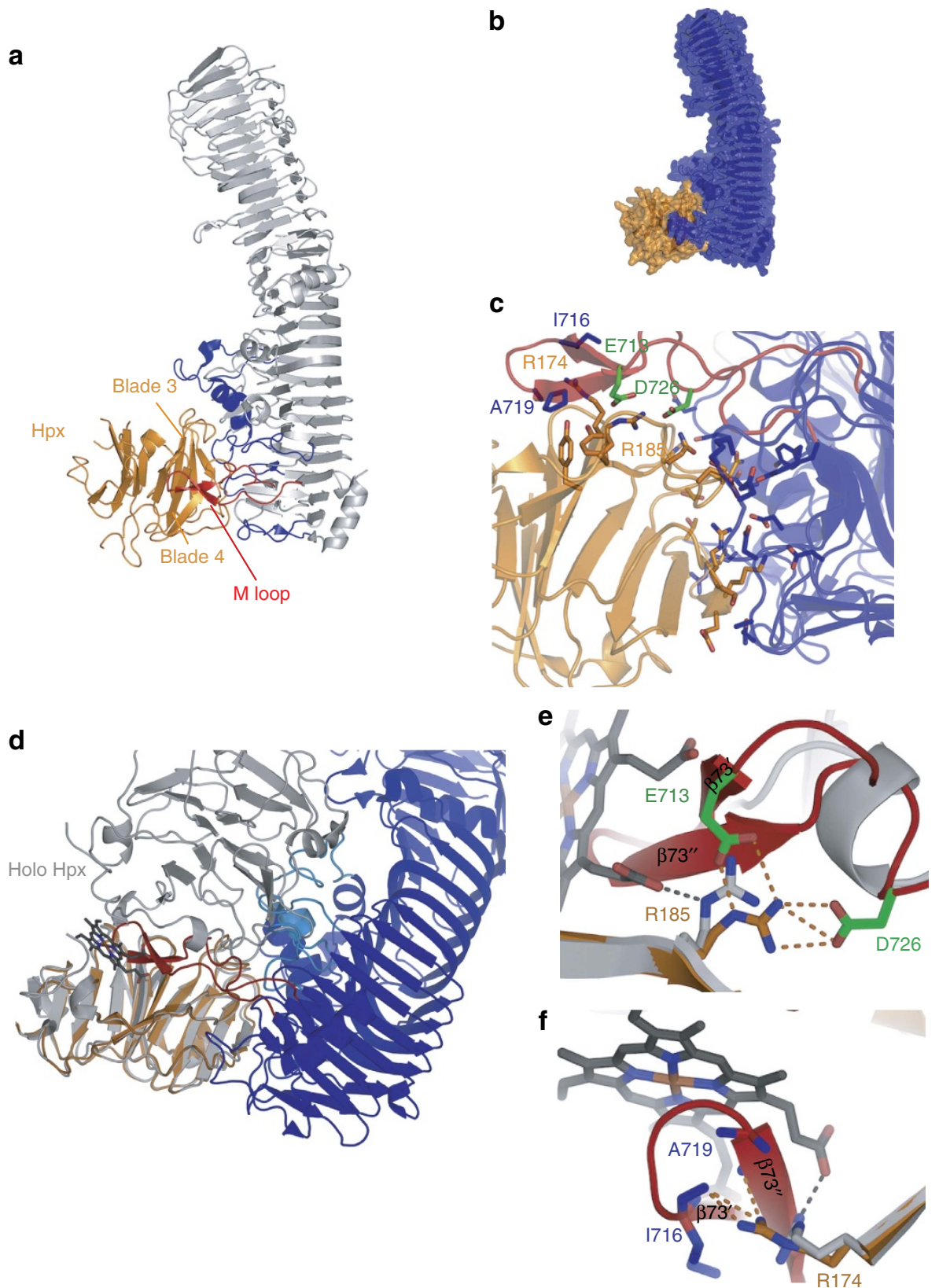

Figure 4 | X-ray structure of the HxuA-NtHpx complex. (a) Cartoon representation of HxuA (silver) in complex with the N-terminal domain of haemopexin (NtHpx; orange). The secondary structure elements in HxuA that provide residues for the interaction with residues from blades 3 and 4 of $\mathrm{NtHpx}$ are coloured blue. The M loop is coloured red. (b) Surface representation of the complex between HxuA (blue) and NtHpx (orange). (c) Enlarged view of the interaction zone. The side chains of residues involved in hydrogen bonds or salt bridges formation is represented in sticks and coloured as in $4 \mathrm{~A}$ side chains of residues Glu713 and Asp726 are shown in green. (d) Superposition of full-length haem-haemopexin (1QHU, in grey) with the structure of the HxuA-NtHpx complex (in blue and orange), showing the potential steric clashes of the C-terminal domain of haemopexin with HxuA (pale blue) and of haem with the M loop of HxuA (red). (e,f) Enlarged view of the region of interaction involving the $\mathrm{M}$ loop together with the reorientation of side chains of Arg174 and Arg185 from haem-haemopexin. The dashes represent atoms located within hydrogen bonding distances.

Key role of the M loop in haem ejection from haem-haemopexin. We constructed three HxuA mutants to test this hypothesis. Their phenotypes were first tested in vivo with respect to their production (Fig. 5a), their ability to bind haemopexin in a dot-blot assay on whole cells (Fig. 5b) and their ability to deliver haem from haem-haemopexin to the $\mathrm{HxuC}$ receptor in the reconstituted system in an E. coli haem auxotroph strain (Fig. 5c). The first, $\mathrm{HxuA}^{\mathrm{DEL}}$, had a deletion of most of the $\mathrm{M}$ loop (residues 711-728, see MM for details). In the $h x u C B A$ context reconstituted in E. coli, this mutant protein was correctly produced at ca. wild-type level (Fig. 5a), and was able to make complexes with haemopexin in vivo, as tested by dot-blot on whole cells using biotinylated haemopexin (Fig. 5b). It did not allow haem acquisition from haemopexin, but had no effect on free haem acquisition (Fig. 5c). The two other mutants were point mutants in Asp726 and Glu713, two residues that establish H-bonds/salt bridges with Arg185 from the N-lobe of haemopexin (one of the residues that stabilize haem propionates in haem-haemopexin) (Fig. 4e). Furthermore, the Arg185 side chain reorientation on interaction with $\mathrm{HxuA}$ is most likely 


\begin{tabular}{|c|c|}
\hline & HxuA-NterHpx \\
\hline \multicolumn{2}{|l|}{ Data collection } \\
\hline Space group & $P 2,2,2$ \\
\hline \multicolumn{2}{|l|}{ Cell dimensions } \\
\hline$a, b, c(\AA)$ & $45.06,348.86,77.35$ \\
\hline$\alpha, \beta, \gamma\left({ }^{\circ}\right)$ & $90.00,90.00,90.00$ \\
\hline Resolution $(\AA)$ & $2.80(2.97-2.80)$ \\
\hline$R_{\text {merge }}$ & $11.5(67.9)$ \\
\hline$l / \sigma l$ & $13.72(2.14)$ \\
\hline Completeness (\%) & $96.3(85.2)$ \\
\hline Redundancy & $7.23(5.15)$ \\
\hline \multicolumn{2}{|l|}{ Refinement } \\
\hline Resolution $(\AA)$ & 2.80 \\
\hline No. reflections & 29,966 \\
\hline$R_{\text {work }} / R_{\text {free }}$ & $0.18 / 0.23$ \\
\hline \multicolumn{2}{|l|}{ No. atoms } \\
\hline Protein & 7,621 \\
\hline Ligand/ion & 28 \\
\hline Water & 192 \\
\hline \multicolumn{2}{|l|}{ B-factors } \\
\hline Protein & 55.70 \\
\hline Ligand/ion & 119.92 \\
\hline Water & 39.55 \\
\hline \multicolumn{2}{|l|}{ R.m.s. deviations } \\
\hline Bond lengths $(\AA)$ & 0.010 \\
\hline Bond angles $\left({ }^{\circ}\right)$ & 1.22 \\
\hline
\end{tabular}

driven by Asp726. Glu713 and Asp726 were replaced by alanine,

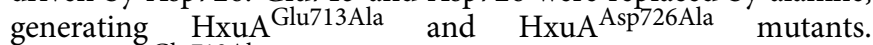
The HxuA Glu713Ala mutant behaved the same as the wild-type in the various tests (Fig. 5a-c), and was not studied further. This was not the case for HxuA Asp726Ala mutant (produced at wild-type level, Fig. 5a), which was strongly affected in its ability to allow growth on haem-haemopexin (Fig. $5 \mathrm{c}$ ) but able to bind haemopexin in vivo (Fig. 5b). Compared with the $\mathrm{M}$ loop deletion mutant, this mutant displayed barely visible, but reproducible, ability to acquire haem from haem-haemopexin (Fig. 5c).

To gain more information on the interaction of those two mutants $\left(\mathrm{HxuA}^{\mathrm{DEL}}\right.$ and $\left.\mathrm{HxuA}^{\text {Asp726Ala }}\right)$, the cysteine-less variants of these mutants were constructed, the corresponding proteins were purified from the supernatant and their interaction with haem-haemopexin tested by both ITC and SEC experiments. ITC experiment (Supplementary Fig. 4) indicated that HxuA ${ }^{\text {DEL }}$ interacted with haem-haemopexin but with different parameters compared with the WT (Supplementary Table 3); a 1:1 complex was formed even though the $\Delta \mathrm{H}$ was substantially lower $\left(-13.0 \mathrm{kcal} \mathrm{mol}^{-1}\right.$ versus $\left.-39.8 \mathrm{kcal} \mathrm{mol}^{-1}\right)$, and the $\mathrm{Ka}$ was also affected $\left(1.7 \times 10^{8} \mathrm{M}^{-1}\right.$ versus $\left.>10^{9} \mathrm{M}^{-1}\right)$. The formation of the complex was also confirmed in SEC experiments (Supplementary Fig. 5) and the complex strikingly contained haem, as evidenced by the absorbance at $410 \mathrm{~nm}$. The absorption spectrum of the complex was very similar to that of haem-haemopexin in the visible part of the spectrum, suggesting that haem did not drastically change environment on interaction (Supplementary Fig. 5b,c). These results pinpoint an essential role for the $\mathrm{M}$ loop in haem ejection from haemopexin, since the interaction with haemopexin takes place without the M loop, but
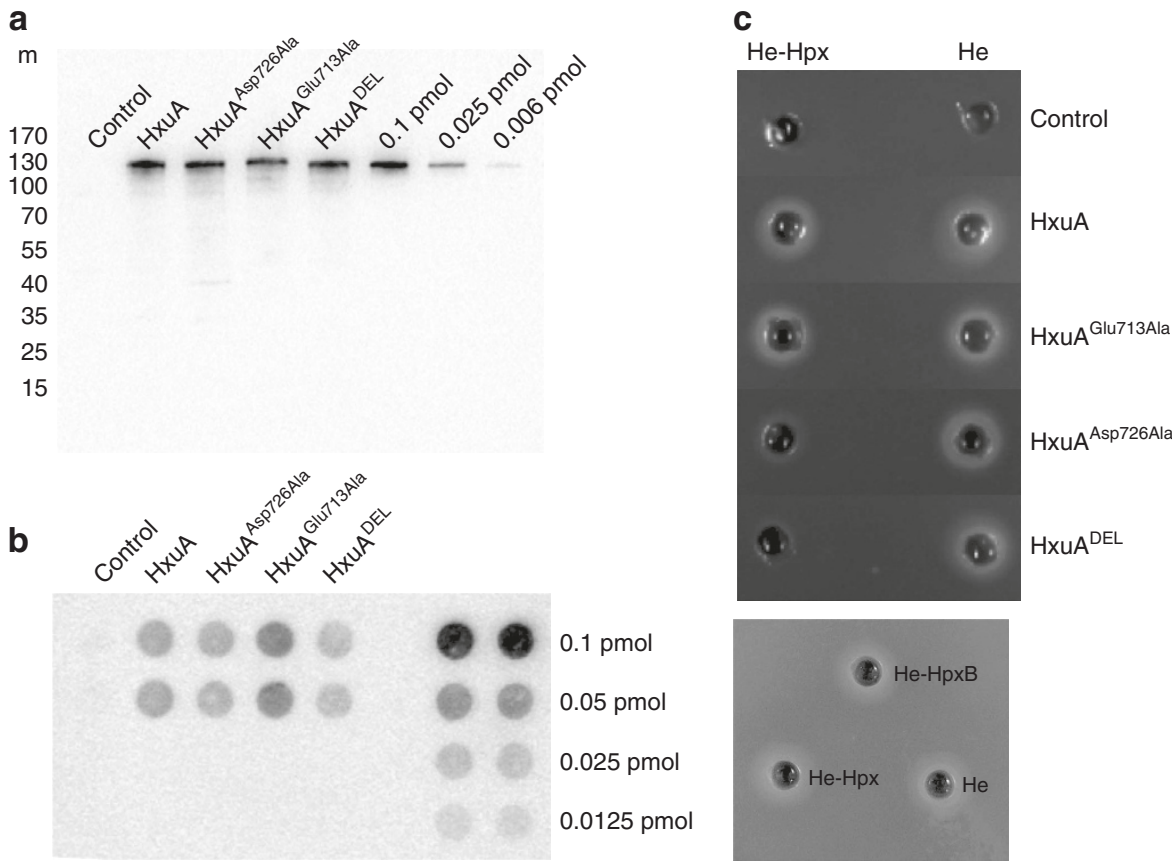

Figure 5 | Phenotypes of HxuA mutants. (a) Amount of HxuA in the various strains, as detected by immunoblotting with anti-HxuA antibodies on whole-cell contents analysed by SDS-PAGE (control, WTHxuA, HxuA Asp726Ala, HxuA Glu713Ala and HxuA ${ }^{\text {DEL }}$ ). The equivalent of $0.05 \mathrm{OD}_{600 \mathrm{~nm}}\left(10^{7}\right.$ cells) was deposited in each lane. Known amounts of purified HxuA were run alongside. The scale on the left $(\mathrm{m})$ represents molecular weight in kDa. (b) Detection of interaction of biotinylated haemopexin with $\mathrm{HxuA}$ and its mutants in whole cells by dot-blot analysis in vivo (control, wild-type, $\mathrm{HxuA}{ }^{\text {Asp726Ala }}$, HxuA Glu713Ala, $\mathrm{Hxu}^{\mathrm{DEL}}$ ). Samples were spotted in duplicate. The equivalent of $0.05 \mathrm{OD}_{600 \mathrm{~nm}}\left(10^{7}\right.$ cells) was deposited in each spot, in duplicate. Known amounts of purified biotinylated haemopexin were run alongside, in duplicate. (c) Analysis of the in vivo activities of HxuA, HxuA ${ }^{D E L}, \mathrm{HxuA}^{\text {Glu713Ala }}$ and HxuA Asp726Ala, in haem acquisition tests from haem and haem-haemopexin. Each well contained $100 \mu \mathrm{l}$ of either $5 \mu \mathrm{M}$ haem (He) or $5 \mu \mathrm{M}$ haem-haemopexin (He-Hpx). The bottom panel shows the same activity test for haem $(\mathrm{He})$, haem-haemopexin $(\mathrm{He}-\mathrm{Hpx})$ and the biotinylated haem-haemopexin (He-HpxB) for WT strain. 
haem ejection is affected. Similar experiments were carried out with the cysteine-less variant of the HxuA Asp726Ala mutant. The mutant protein also formed stoichiometric complexes with haem-haemopexin (Supplementary Fig. 4). Both $\Delta \mathrm{H}$ and $\mathrm{Ka}$ were affected compared with the wild type (Supplementary Table 3) ( $-12.8 \mathrm{kcal} \mathrm{mol}^{-1}$ versus $-39.8 \mathrm{kcal} \mathrm{mol}^{-1}$, and $2.9 \times 10^{6} \mathrm{M}^{-1}$ versus $>10^{9} \mathrm{M}^{-1}$, respectively). After gel filtration, the purified complex still showed absorbance in the Soret region, indicating that haem was present, and the absorption spectrum was not significantly different from that of haem-haemopexin, except for a 2-nm red-shift of the Soret band maybe indicative of a slight distorsion of the haem geometry on interaction (Supplementary Fig. 5b,c). There was a detectable reduction in the ratio of the $414 \mathrm{~nm} / 280 \mathrm{~nm}$ absorbance as compared with the HxuA ${ }^{\mathrm{DEL}}$-haem-haemopexin complex, potentially indicating partial release of haem on complex formation (Supplementary Fig. 5), which could explain the minute but detectable growth observed with this mutant. These results indicate that a single residue, Asp726 (that is conserved in all $\mathrm{HxuAs}^{19}$ ), is essential for haem acquisition from haem-haemopexin. Finally, whereas the $\Delta H$ 's of interaction of the two HxuA mutants with haem-haemopexin are quite similar (Supplementary Table 3), they are quite different with haemopexin (Supplementary Table 3). This might indicate that, as there is almost no haem release from haem-haemopexin by the HxuA Asp726Ala mutant, the polar interactions between haem-haemopexin and HxuA mutants are similar. On the other hand, with haemopexin, the $M$ loop from the HxuA Asp726Ala mutant could establish close to wild-type contacts with haemopexin, which obviously could not form with the $\mathrm{HxuA}^{\mathrm{DEL}}$ mutant.

It is therefore likely that, at least, a two-step process occurs. Initially, haemopexin would be captured by HxuA, without gross modification of the haemopexin structure and haem ligation; this might correspond to the state stabilized by the Asp726 mutant. Steric clashes between the C-terminal domain of haemopexin and HxuA together with the insertion of the M loop in the cleft between the two domains, up to the interaction of Asp726 from HxuA M loop with specific haemopexin residues, would then trigger haem release and its capture by the $\mathrm{HxuC}$ receptor.

\section{Discussion}

Haem acquisition is a major route by which pathogens/ commensals take up iron. In most cases, it depends on bacterial haemophores that recognize either haem and/or the host haemoprotein ${ }^{20}$. The purpose of haemophores is twofold, first to allow a very efficient haem/haemoprotein capture, second to ultimately trigger haem transfer to a membrane bound component that actively transports haem across the bacterial membrane. Two haemophores classes have been particularly well studied, the HasA (Gram-negative) and NEAT (Gram-positive) types. In both cases, interaction with haem and haem transfer to the membrane bound transporter have been extensively studied ${ }^{21-23}$, but the precise mechanism of interaction with the host haemoprotein leading to haem extraction are not understood. HxuA defines a new class of haemophores that promote haem release from haemopexin without having a detectable affinity for haem $^{12}$.

HxuA belongs to the same class of TpsA molecules as HMW1A. It folds as a right-handed $\beta$-helix, as expected for a TpsA protein ${ }^{24}$. The $\mathrm{N}$-terminal secretion domain terminates with a $\alpha$-helix not predicted from the primary sequence. Together with a strand swapping in one of the parallel $\beta$-sheets $(\beta 40-\beta 41)$, this helix introduces a small bend and a change of register in the whole molecule. Whether this is a conserved feature of TpsA molecules or specific to HxuA remains to be determined. FHA and HMW1A display extended signal peptides characteristic of the TPS pathway. This is not the case for HxuA, maybe indicating differences in the secretion mechanism. The structure itself does not allow further conclusions.

The various TpsA proteins function in different ways. They range from adhesins (FHA from $B$. pertussis, HMW1A from H. influenzae $)^{25-27}$ to pore-forming toxins ${ }^{28-30}$ to proteases ${ }^{31}$. HxuA defines a specific class of haemophores. Its function requires the precise binding of haemopexin so as to release its bound substrate (haem). This is ensured by the very long insertions, which protrude from the $\beta$ helical scaffold in the C-terminal part and form the haemopexin binding site.

The last 69 residues of $\mathrm{HxuA}$ are not seen in the $\mathrm{X}$-ray structure. Yet, the mass spectrometry data indicate that this part of the molecule is not cleaved, at least for a substantial proportion of the molecules, indicating that this C-terminal part is likely disordered. Like HMW1A, HxuA has two cysteine residues at the C-terminus. In HMW1A, these somehow anchor the molecule to the outer membrane. It is believed that, like in the HMW1A case, the extreme C-terminus of HxuA with this disulfide bridge resides in the periplasm (residues 854-884) and that the preceding sequence (residues $824-853$ ) resides in the HxuB barrel as an $\alpha$-helix. FhaC, the TpsB of the FHA system, can accomodate an $\alpha$-helix in its barrel ${ }^{32}$. It is therefore likely that a very short linker (residues 819-823) connects the end of our structure (residue 818) and the helix inserted in the $\mathrm{HxuB}$ barrel, putting the HxuA molecule very close to the cell surface.

A plausible scenario for haem acquisition would be as follows: HxuA lies on the cell surface with its C-terminal amphipatic helix directed towards the outer membrane, and the protruding loops from the C-terminal domain directed towards the extracellular milieu. Haemopexin capture takes place at the C-terminal end of HxuA by a highly polar/charged interface. Rearrangement of the $M$ loop in the cleft between the two MMP domains of haemopexin, could trigger additional conformational changes in haemopexin, weakening haem binding and leading to haem release and its capture by the $\mathrm{HxuC}$ receptor. Several points support this model. First, there is one highly negatively charged patch at the HxuA surface (Asp563, Asp685, Asp738 from different loops at the HxuA surface) that comes in close contact with a positive patch on Hpx (Lys155, Arg157). Second, M loop movement from its initial position to its position in the complex, might also be driven by ionic interactions between the positively charged haemopexin and the negatively charged $M$ loop. This is coherent with the very different interactions measured between haemopexin and wild-type HxuA on the one hand, and between haemopexin and HxuA ${ }^{\mathrm{DEL}}$ on the other hand. This is also coherent with the similar interactions measured between haemopexin and wild-type HxuA on the one hand, and between haemopexin and HxuA Asp726Ala on the other hand. The water molecules that lie at the interface of the two haemopexin domains have been envisioned as key players in relative movements of both domains leading to haem release ${ }^{8}$, and it is obvious that $\mathrm{M}$ loop positioning at the contact of the $\mathrm{N}$-domain of haemopexin will also lead to water molecules displacement. In the hepatocyte, although there is debate about the identification of the genuine haemopexin receptor ${ }^{33,34}$, receptor driven endocytosis leads to haem-haemopexin internalization and haem release probably occurs in endocytic vacuoles at a $\mathrm{pH}$ below 5 , likely associated with histidine protonation. This is not the case for haem release from haem-haemopexin by HxuA, which leads to question the relative contribution of the different elements to haem binding, respectively histidine residues, 
hydrophobic stacking to the pyrrole ring from several residues and propionate stabilization by positively charged residues.

Regarding the late steps of this process, it is possible that the $\mathrm{HxuC}$ receptor might interact with either HxuA or the HxuA-haemopexin complex to efficiently capture haem released from haemopexin. We have previously shown that HxuAdm (the cysteine-less variant of HxuA) is inactive in the context of the reconstituted system in E. coli, despite being perfectly able to discharge haem from haem-haemopexin in vitro. Our current hypothesis is that function of the $\mathrm{Hxu}$ system requires dissociation of haemopexin from the HxuA molecule, once haem from haem-haemopexin has been taken up by the HxuC receptor. This dissociation step would require energy transduced by the TonB complex, and hence a direct interaction with either TonB or HxuC is likely to exist.

In contrast to the N. meningitidis $\mathrm{TbpA} / \mathrm{TbpB}$ receptor, which is exquisitely specific for human transferrin ${ }^{35}$, the Hxu system from $H$. influenzae can accept haemopexins at least from human and rabbit $^{36}$. When the structures of human and rabbit haemopexin ( $82 \%$ sequence identity) were compared, all haemopexin residues interacting with HxuA residues by their side chains are conserved, with one exception. Also, for TbpA/TbpB-transferrin, it is very likely that, TbpB fulfills a shielding function to avoid Fe leakage towards the extracellular medium as well as discriminating towards Fe-transferrin. In the HasA-HasR-haem case, haem still has access to its binding site on HasR from the extracellular medium ${ }^{22,37,38}$. This is not yet known for the HxuA-haemopexin-HxuC system. In both the HasA-HasR and TbpA-TbpB-transferrin systems, haem/iron release is carried out by extracellular loops of the TonBdependent receptor 22,39 , which in turn releases the discharged carrier protein. This is clearly different from the Hxu system, where haem release from haemopexin does not involve the TonB-dependent HxuC receptor. As compared with the strategies used by Gram-positive bacteria, examplified by the Isd system from $S$. aureus where the initial capture of haem from haemoglobin is insured ${ }^{40}$ by NEAT domains containing IsdB or IsdH. In both cases, a NEAT domain having no affinity for haem but for haemoglobin, is linked via a linker domain that allows the correct positioning of a second NEAT domain having affinity for haem, and very little affinity for haemoglobin. Haem is then transferred through several NEAT domains by a so-called hand-clasp mechanism ${ }^{23}$ to the permease.

Finally, it is also possible that $\mathrm{HxuA}$, besides its function in preventing haem binding to haemopexin, also prevents haemopexin binding to its specific hepatocyte receptor, as it partially covers an epitope (residues 120-142), essential for this interaction ${ }^{41}$.

\footnotetext{
Methods

Protein expression and purification. Full-length $H x u A$. HxuA was expressed in $E$. coli BL21 transformed with a pBAD24 derivative harbouring the $h x u C B A$ operon. The two last cysteine codons of $h x u A$ were respectively changed to serine, to obtain a higher release of HxuA into the culture supernatant ${ }^{12}$

The strain was grown in a 4-1 fermentor at the Institut Pasteur Protein Production Facility, in medium density medium, with $80 \%$ pO2 throughout at $37^{\circ} \mathrm{C}$. At an $\mathrm{OD}_{600 \mathrm{~nm}}$ of ca. $4-5$, the culture was induced with arabinose $\left(20 \mu \mathrm{g} \mathrm{ml}^{-1}\right)$ for $\sim 150 \mathrm{~min}$, up to a maximum $\mathrm{OD}_{600 \mathrm{~nm}}$ of 30 . After quick cooling to ca. $10^{\circ} \mathrm{C}$, the culture was centrifuged $(20 \mathrm{~min}$ at $10,000 \mathrm{~g})$. The supernatant was withdrawn and quickly supplemented with EDTA (10 mM final), Tris base ( $1 \mathrm{mM}$ final) and ammonium sulfate ( $80 \%$ saturation). After incubation at $4{ }^{\circ} \mathrm{C}$ for $1 \mathrm{~h}$, the supernatant was centrifuged $(45 \mathrm{~min}$ at $12,000 \mathrm{~g})$. The resulting pellet was resuspended in $20 \mathrm{mM}$ Tris- $\mathrm{HCl} \mathrm{pH} 7.5,150 \mathrm{mM} \mathrm{NaCl}, 10 \mathrm{mM}$ EDTA and dialyzed against the same buffer overnight. The supernatant was centrifuged for $3 \mathrm{~h}$ (at $200,000 \mathrm{~g}$ ) at $4{ }^{\circ} \mathrm{C}$, to eliminate insoluble material, filtered through a $0.2-\mu \mathrm{m}$ filter and concentrated using an Amicon-Ultra 15 Ultracel $50 \mathrm{~K}$ (50 kDa cutoff) device down to a volume of ca. $30 \mathrm{ml}$. The retentate was filtered through a $0.2-\mu \mathrm{m}$ filter and a SEC step (Superdex $200 \mathrm{pg} 26 \times 60$ ) was run in the same buffer and $4 \mathrm{ml}$ fractions collected. Fractions of interest (as analysed by SDS-polyacrylamide gel
}

electrophoresis (SDS-PAGE)) were pooled, concentrated down to $<1 \mathrm{ml}$, then the buffer was exchanged for $20 \mathrm{mM}$ Tris- $\mathrm{HCl} \mathrm{pH} \mathrm{9.0,15} \mathrm{mM} \mathrm{NaCl}, 1 \mathrm{mM}$ EDTA and finally loaded on a monoQ HR 10-100 column. Sample was eluted with a $\mathrm{NaCl}$ gradient from 15 to $500 \mathrm{mM}$ in the same buffer over 15 column volumes. Fractions $(0.5 \mathrm{ml})$ were collected, analysed by SDS-PAGE and pure fractions of HxuA were pooled, concentrated up to $35-40 \mathrm{mg} \mathrm{ml}^{-1}$ for immediate crystallization trials, or kept frozen at $-80^{\circ} \mathrm{C}$ until use. The same protocol was employed for HxuA mutants. HxuA concentration was estimated after amino acid analysis of a representative preparation at the Unité de Chimie des Biomolécules (Institut Pasteur). The amino acid composition was in excellent agreement with HxuA composition and led to an estimated molar extinction coefficient of $50,990 \mathrm{M}^{-1} \mathrm{~cm}^{-1}$ instead of the calculated one of $44,500 \mathrm{M}^{-1} \mathrm{~cm}^{-1}$. The same sample analysed by mass spectrometry (Supplementary Fig. 2) gave a mass of 96,467.6094 (theoretical mass: 96,469.6926).

Rabbit haemopexin. Hpx was purified from $500 \mathrm{ml}$ of rabbit serum (Biosera minimal haemolysis), by a modification of the protocol described by Morgan ${ }^{41}$ Briefly, phenyl methyl sulfonyl fluoride (at $0.1 \mathrm{mM}$ final) was added to thawed rabbit serum. $62.5 \mathrm{ml}$ of $1.8 \mathrm{M}$ perchloric acid was slowly added under agitation at $4{ }^{\circ} \mathrm{C}$, and agitation was continued for a further $10 \mathrm{~min}$. The solution was centrifuged $(20 \mathrm{~min}$ at $10,000 \mathrm{~g})$ and the supernatant was adjusted to $\mathrm{pH} 8.0$ with $10 \mathrm{~N} \mathrm{NaOH} .50 \%$ (w/v) PEG 3400 (Polyscience) was added to a final concentration of $15 \%$. After stirring for $1 \mathrm{~h}$ at $4{ }^{\circ} \mathrm{C}$ and centrifugation $(20 \mathrm{~min}$ at $10,000 \mathrm{~g}$ ), the supernatant was adjusted with solid PEG 3400 to a final concentration of $25 \%$ (w/v), and stirred $1 \mathrm{~h}$ at $4^{\circ} \mathrm{C}$. After centrifugation $(20 \mathrm{~min}$ at $10,000 \mathrm{~g})$, the pellet was resuspended in $20 \mathrm{mM}$ Tris- $\mathrm{HCl} \mathrm{pH} \mathrm{8.0,} \mathrm{concentrated} \mathrm{using} \mathrm{a} \mathrm{Millipore}$ centrifugal device (Amicon-Ultra 15 Ultracel $10 \mathrm{~K}$ ), washed twice with the same buffer and finally with $20 \mathrm{mM}$ MES-NaOH pH 6.0. The sample was loaded on a 20 -ml S-sepharose column equilibrated with $20 \mathrm{mM} \mathrm{MES-NaOH} \mathrm{pH} \mathrm{6.0,} \mathrm{and}$ eluted with a linear gradient of $\mathrm{NaCl}$ from 0 to $1 \mathrm{M}$ in the same buffer over 20 column volumes. Four millilitre fractions were collected and haemopexin eluted at $\sim 0.2 \mathrm{M} \mathrm{NaCl}$. The fractions of interest were concentrated as above and the buffer was exchanged for $20 \mathrm{mM}$ Tris- $\mathrm{HCl} \mathrm{pH} \mathrm{7.5.} \mathrm{The} \mathrm{sample} \mathrm{was} \mathrm{then} \mathrm{loaded} \mathrm{onto} \mathrm{a}$ mono Q HR10-100 column equilibrated with $20 \mathrm{mM}$ Tris- $\mathrm{HCl} \mathrm{pH} \mathrm{7.5,} \mathrm{and} \mathrm{eluted}$ with a linear $\mathrm{NaCl}$ gradient up to $1 \mathrm{M}$ in the same buffer over 15 column volumes. The fractions $(4 \mathrm{ml})$ were collected, pooled and then concentrated before being run on a SEC column (Superdex $200 \mathrm{pg}, 26 \times 60$ ), which was equilibrated in $20 \mathrm{mM}$ Tris- $\mathrm{HCl} \mathrm{pH} \mathrm{7.5,} 150 \mathrm{mM} \mathrm{NaCl}$ and $10 \mathrm{mM}$ EDTA. Four millilitre fractions of pure haemopexin, as judged from Coomassie blue staining and haem binding ability, were collected, pooled, concentrated and kept at $-80^{\circ} \mathrm{C}$ until use. The yield was $\sim 20 \mathrm{mg}$ haemopexin starting from $500 \mathrm{ml}$ of serum.

$N$ - and C-terminal sub-domains of haemopexin. Haemopexin $(1 \mathrm{ml}$ at $30 \mu \mathrm{M}$, as estimated from the theoretical molar extinction coefficient of $118,090 \mathrm{M}^{-1} \mathrm{~cm}^{-1}$ at $280 \mathrm{~nm})$, was digested for $1 \mathrm{~h}$ at $37^{\circ} \mathrm{C}$ with trypsin $\left(0.2 \mu \mathrm{l}, 1 \mathrm{mg} \mathrm{ml}^{-1}\right)$. The reaction was stopped with phenyl methyl sulfonyl fluoride ( $1 \mathrm{mM}$ final). Under these conditions the digestion was not complete, $\sim 1 / 3$ of the initial haemopexin was still intact and two smaller fragments were observed. Those two smaller fragments, respectively, corresponding to the $\mathrm{N}$-terminal and $\mathrm{C}$-terminal domains of haemopexin were further purified by two successive steps of chromatography, first on a mono S HR10-100 column, then by SEC. N-terminal sequencing indicated that haemopexin was cleaved in the linker after Arg 214.

Purification of the complexes. All complexes were purified by mixing a ca. 1.2-fold molar excess of either He-Hpx or the NtHpx fragment with either HxuA or its mutants, and running a SEC column (Superdex $200 \mathrm{pg}, 26 / 60$ ) equilibrated with $20 \mathrm{mM}$ Tris- $\mathrm{HCl} \mathrm{pH} 7.5,150 \mathrm{mM} \mathrm{NaCl}, 10 \mathrm{mM}$ EDTA.

Crystallization. Initial screening of crystallization conditions was carried out for HxuA alone and for HxuA in complex with the N-terminal domain of rabbit haemopexin, using the vapour diffusion method with a Mosquito nanolitredispensing system (TTP Labtech). Sitting drops were set up using $400 \mathrm{nl}$ of a 1:1 mixture of each sample protein and crystallization solutions (672 different commercially available conditions) equilibrated against a $150 \mu \mathrm{l}$ reservoir in multiwell plates (Greiner Bio-One). The crystallization plates were stored at $18^{\circ} \mathrm{C}$ in a RockImager1000 (Formulatrix) automated imaging system to monitor crystal growth. Manual optimization was performed in Limbro plates by the hanging-drop method. The best crystals of HxuA were obtained at a temperature of $18^{\circ} \mathrm{C}$ by mixing an equal amount of protein solution $\left(35 \mathrm{mg} \mathrm{ml}^{-1} \mathrm{HxuA}\right)$ and reservoir solution (0.2 $\mathrm{M} \mathrm{MgCl}_{2}, 0.1 \mathrm{M}$ HEPES, pH 7.5, 30\% w/v PEG 400). Crystals appeared after 2-4 days. For the complex HxuA-NtHpx, crystals were obtained by the same technique using the protein solution at a concentration of ca. $50 \mathrm{mg} \mathrm{ml}^{-1}$ mixed with the following reservoir solution: $100 \mathrm{mM}$ citric acid pH 5.60, $200 \mathrm{mM}$ ammonium sulfate and 25\% w/v PEG 4000. The time needed for crystals to appear was 1-2 weeks.

Single crystals of HxuA were flash-cooled in liquid nitrogen, using the mother crystallization liquid as cryoprotectant. For HxuA-NtHpx crystals, a mixture of $50 \%$ Paratone- $\mathrm{N}$ and $50 \%$ paraffin oil was used as cryoprotectant.

Structure determination and refinement. X-ray diffraction data from single crystals $\left(\right.$ at $\left.-173^{\circ} \mathrm{C}\right)$ for HxuA and HxuA-NtHpx were collected on beamline 
PROXIMA-1 at the Synchrotron SOLEIL (Saint-Aubin, France). Data were processed with XDS (ref. 42) and scaled with XSCALE, from the XDS package.

The structure of HxuA was solved by the single isomorphous replacement with anomalous signal method. A first data set was collected on a native crystal at a wavelength of $\lambda=0.97918 \AA$. An Europium derivative was prepared by soaking a native crystal overnight in a solution containing Eu-(tris(pyridine-2,6dicarboxylate) or DPA) ${ }_{3}$ lanthanide purchased from NatX-ray (Grenoble, France). A second data set was then collected on this derivative, at the energy corresponding to the peak of the Eu K-edge ( $48.5200005 \mathrm{keV}, \lambda=1.775520 \AA)$, as determined by an X-ray fluorescence scan. Two Eu sites were located using the programme SHELXD (ref. 43). Phasing was done with the programme Phaser ${ }^{44}$, and initial automatic model building proceeded with ARP/WARP (ref. 45). The model was then improved through iterative cycles of manual model adjustment and building with COOT (ref. 46). Final refinement was performed with autoBUSTER (ref. 47). Residues Ser1, Gly117-Gly121, Pro273, Lys274, Gly344-Thr349, Phe565-Gly571, Ile723-Asn728, Arg785-Lys793 and Ala818-Gln884 were not seen in the electron density map, and were not incorporated into the model.

The structure of HxuA in complex with the $\mathrm{N}$-terminal domain of haemopexin was solved by molecular replacement with Phaser ${ }^{44}$ by using the structure of native HxuA and the N-terminal domain of haemopexin (PDB entry code 1QHU) as a template. Here also, the model was further improved through iterative cycles of manual model adjustment and building with COOT, and final refinement was performed with autoBUSTER. Residues Ser1, Ala2, Gly119-Gly121, Asp195, Asn196, Arg220, Pro273, Lys274, Val816-Gln884 from HxuA and residues Val1-Val23, Gly58-Leu62, Gly81, His82, Glu99-Val104 and Pro209-Arg214 from $\mathrm{NtHpx}$ were not seen in the electron density map and were not incorporated into the model.

All models were validated through the Molprobity server (http://molprobity. biochem.duke.edu $)^{48}$. Figures were generated and rendered with Pymol (the PyMOL Molecular Graphics System, Version 1.5, Schrödinger, LLC.). The crystal parameters, data statistics and final refinement parameters are shown in Tables 1 and 2. Ramachandran statistics indicate that for HxuA 96.5\% (742/769) of all residues are in favoured (98\%) regions, and 100.0\% (769/769) of all residues are in allowed (>99.8\%) regions. Regarding HxuA in complex with $\mathrm{N}$-ter haemopexin, 94.7\% (908/959) of all residues are in favoured (98\%) regions, and $99.7 \%(956 / 959)$ of all residues are in allowed (>99.8\%) regions. Atomic coordinates and structure factors have been deposited in the Brookhaven Protein Data Bank under the accession numbers 4RM6 for HxuA and 4RT6 for HxuA in complex with $\mathrm{N}$-ter haemopexin.

Isothermal titration calorimetry. Proteins were dialyzed against $20 \mathrm{mM}$ Na-phosphate buffer $\mathrm{pH} 7.0$; the concentration of haemopexin and its fragments in the syringe was $\sim 100 \mu \mathrm{M}$ and the HxuA concentration in the titration cell was $\sim 10 \mu \mathrm{M}$. ITC experiments were performed at $25^{\circ} \mathrm{C}$ using the high precision VP-ITC system (MicroCal, GE Healthcare) ${ }^{49}$. In total, 35 individual $7 \mu$ aliquots were injected from the $250 \mu \mathrm{l}$ syringe into the $1.41 \mathrm{ml}$ titration cell, with a 6 -min interval between injections, unless otherwise stated. Heat signals were corrected for the respective heat of dilution and normalized to the amount of compound injected. The enthalpy of binding $(\Delta \mathrm{H})$, affinity constant $(\mathrm{Ka})$ and molar binding stoichiometry $(n)$ were directly obtained from the titration curve fitted using the single-site binding model of the Origin7software (OriginLab), even though there might be heterogeneity in the HxuA binding sites. HxuA concentrations were measured according to the experimentally determined molar extinction coefficient. Given the protein concentration in the titration cell $(\sim 10 \mu \mathrm{M})$, the size of the injection and the limitation of the technique itself ( $\mathrm{Ka} \mathrm{x}$ substrate concentration between 1 and 1,000$)^{49}$, the absolute value of the Ka determined should be taken with caution. Besides, as there are conformational changes in the interactants on interaction the values should be taken as apparent Ka's.

TEM and image processing. For negative stain TEM, the protein solution was diluted at $10-20 \mu \mathrm{g} \mathrm{ml}^{-1}$ in PBS buffer. Immediately afterwards, $4 \mu \mathrm{l}$ aliquot was adsorbed onto glow discharged carbon film coating copper EM grids, washed with three droplets of pure water and subsequently negatively stained with $2 \%(\mathrm{w} / \mathrm{v})$ uranyl-acetate. The prepared grids were imaged using a Philips CM10 TEM (FEI Company, Eindhoven, the Netherlands) operating at $80 \mathrm{kV}$. The images were recorded by the $2 \mathrm{k} \times 2 \mathrm{k}$ side-mounted Veleta CCD camera (Olympus, Germany) at a magnification of $\times 130,000$. The pixel size at the sample level was $3.7 \AA$.

Image processing was carried out using the EMAN2 software package ${ }^{50}$. The images were contrast transfer function corrected and particle projections were semi-automatically selected. The e2refine2d programme was used to classify the particle projections. This programme produces reference-free class averages from a population of mixed, unaligned particle projections. The representative class averages with the best signal-to-noise ratio were selected and gathered in a gallery.

Mutant construction. Plasmid phxuCBA was amplified with the following three oligonucleotides couples:

revglu 5'-CCAGGCGCGCCATTAATGATCACTGCTTTCCCAGTTCTTCC-3', forglu $5^{\prime}$-ATGGCGCGCCTGGCTCAATTGATAATGATG-3', revasp 5'-CCAGGCGCGCCATTAATGATCACTTCTTTCC-3', forasp $5^{\prime}$-AATGGCGCGCCTGGCTCAATTGATAATGCTGCGAATAT TGCC- $3^{\prime}$,

revdel 5'-CCAGGGCCCAGTTCTTCCTCCAGTGTTAATTGCGG- ${ }^{\prime}{ }^{\prime}$ and

fordel 5'-AATGGGCCCATTGCCAATATGGCATTTACTATTGG-3'.

In bold are shown the sequences corresponding to the introduced restriction sites, AscI (HxuA Glu713Ala and HxuA Asp726Ala mutants), PspOMI (HxuA ${ }^{\mathrm{DEL}}$ mutant). After amplification, the PCR reaction was digested with DpnI, and the PCR product digested either with AscI (HxuA Glu713Ala and HxuA Asp726Ala mutants) or PspOMI (HxuA ${ }^{\mathrm{DEL}}$ mutant $)$ and self-ligated before transformation into E. coli XL1-Blue strain. After checking for the presence of the desired mutation by sequencing, a 900-bp BsrGI-SphI fragment encompassing the desired mutation was recloned into an otherwise wild-type $h x u C B A$ background. To obtain the $\mathrm{dm}$ variant of the mutants (that is, cysteine-less at the C-terminus), the 500-bp Bpu10ISphI fragment from the $\mathrm{dm}$ variant was exchanged with that of the previously constructed plasmids (Supplementary Fig. 6).

HxuA detection in whole cells. Polyclonal antibodies directed against full-length purified HxuA were raised in rabbits at the Institut Pasteur facility in 2011, in compliance with good practice in use at that time. The serum was used in western blots at a 1/2000 dilution after depletion of unspecific antibodies from the serum by adsorption on a cell lysate from a strain not expressing HxuA. Briefly, $20 \mu \mathrm{l}$ of serum were mixed for $1 \mathrm{~h}$ at $4{ }^{\circ} \mathrm{C}$ with $1 \mathrm{ml}$ of a cell lysate obtained by sonication of the equivalent of $100 \mathrm{ml}$ of an overnight culture resuspended in PBS. After centrifugation $(15,000 \mathrm{~g}$ for $15 \mathrm{~min})$, the supernatant represents the 'depleted serum'. Blotting itself after gel electrophoresis was carried out for $2 \mathrm{~h}$ in a semi-dry apparatus, at $1 \mathrm{~mA} \mathrm{~cm}^{-2}$ and $12 \mathrm{~V}$ maximum on Protran BA 83 nitrocellulose membrane. Whole cultures were TCA-precipitated (10\%) and the samples were run on commercial gels (mini Protean TGX 4-15\% gradient gels from BioRad). The equivalent of $0.05 \mathrm{OD}_{600 \mathrm{~nm}}$ (ca. $10^{7}$ cells) was deposited on each lane.

Haemopexin biotinylation and dot-blot assays on whole cells. Full-length haemopexin was biotinylated with the EZ-LINK Sulfo-PEG4-Biotinylation Kit from Pierce, following the kit instructions. There were 4,5 biotin molecules/haemopexin molecule, as determined using the HABA/avidin ((2- [4'-hydroxybenzeneazo] benzoic acid)) reagent present in the kit. After haem-loading, this biotinylated haem-haemopexin was fully functional in haem acquisition tests in vivo (Fig. $5 \mathrm{c}$, bottom). This biotinylated haemopexin at $0.1 \mu \mathrm{M}$ was subsequently used in dot-blot assays on whole-cells expressing HxuA and its mutants in a protocol similar to that used to detect HasA-HasR interaction ${ }^{51}$. Briefly, cells from various strains were grown in the presence of arabinose $\left(40 \mathrm{mgl}^{-1}\right)$ to induce the expression of the $h x u$ genes up to an $\mathrm{OD}_{600 \mathrm{~nm}}$ of 1 and $0.05 \mathrm{ml}$ were spotted in duplicate for each culture on Protran BA 83 nitrocellulose membrane. After membrane drying and saturation with TBST $(10 \mathrm{mM}$ Tris-HCl pH $8.0,150 \mathrm{mM}$ $\mathrm{NaCl}, 0.05 \%$ Tween-20) containing $0.5 \%$ skimmed milk, the membrane was incubated for $1 \mathrm{~h}$ with $0.1 \mu \mathrm{M}$ biotinylated haemopexin. Bound haemopexin was revealed using High Sensitivity Streptavidin HRP from Pierce (ref 21130).

Biological activity tests. The strain E. coli C600 4 hemA $A$ tonB $\Delta$ exbBD(ptonBHi) was transformed with the wild-type and mutant phxuCBA plasmids, and tested for overnight growth with haem and haem-haemopexin as a haem source as described previously ${ }^{12}$. Briefly, cells were grown up to an $\mathrm{OD}_{600 \mathrm{~nm}}$ of 1 and $0.1 \mathrm{ml}$ of cells were mixed with $3.5 \mathrm{ml}$ of molten soft-agar $(0.6 \%$ in lysogeny broth medium) and poured on the top of a petri dish containing lysogeny broth agar with the appropriate antibiotics and arabinose $\left(40 \mathrm{mgl}^{-1}\right)$ to induce the expression of the $h x u$ genes. Wells were made with the large end of a Pasteur pipette after solidification of the top agar, and filled with $100 \mu \mathrm{l}$ of the various haem sources, at $5 \mu \mathrm{M}$. Growth around the wells was scored after overnight incubation at $37^{\circ} \mathrm{C}$.

\section{References}

1. Noinaj, N., Guillier, M., Barnard, T. J. \& Buchanan, S. K. TonB-dependent transporters: regulation, structure, and function. Annu. Rev. Microbiol. 64, 43-60 (2010).

2. Cassat, J. E. \& Skaar, E. P. Iron in infection and immunity. Cell Host Microbe 13, 509-519 (2013).

3. Contreras, H., Chim, N., Credali, A. \& Goulding, C. W. Heme uptake in bacterial pathogens. Curr. Opin. Chem. Biol. 19, 34-41 (2014).

4. Wong, S. M. \& Akerley, B. J. Genome-scale approaches to identify genes essential for Haemophilus influenzae pathogenesis. Front. Cell. Infect. Microbiol. 2, 23 (2012).

5. Seale, T. W. et al. Complex role of hemoglobin and hemoglobin-haptoglobin binding proteins in Haemophilus influenzae virulence in the infant rat model of invasive infection. Infect. Immun. 74, 6213-6225 (2006).

6. Hanson, M. S., Pelzel, S. E., Latimer, J., Muller-Eberhard, U. \& Hansen, E. J. Identification of a genetic locus of Haemophilus influenzae type $b$ necessary for the binding and utilization of heme bound to human hemopexin. Proc. Natl Acad. Sci. USA 89, 1973-1977 (1992). 
7. Smith, A. Mechanisms of Cytoprotection by Hemopexin. in Handbook of Porphyrin Science Vol. 15 (eds Kadish, K. M., Smith, K. M. \& Guilard, R.) 217-355 (World Scientific, 2011).

8. Paoli, M. et al. Crystal structure of hemopexin reveals a novel high-affinity heme site formed between two b-propeller domains. Nat. Struct. Biol. 6, 926-931 (1999).

9. Cope, L. D. et al. The $100 \mathrm{kDa}$ haem:haemopexin-binding protein of Haemophilus influenzae: structure and localization. Mol. Microbiol. 13, 863-873 (1994).

10. Cope, L. D., Yogev, R., Muller-Eberhard, U. \& Hansen, E. J. A gene cluster involved in the utilization of both free heme and heme:hemopexin by Haemophilus influenzae type b. J. Bacteriol. 177, 2644-2653 (1995).

11. Jacob-Dubuisson, F., Locht, C. \& Antoine, R. Two-partner secretion in Gram-negative bacteria: a thrifty, specific pathway for large virulence proteins. Mol. Microbiol. 40, 306-313 (2001).

12. Fournier, C., Smith, A. \& Delepelaire, P. Haem release from haemopexin by HxuA allows Haemophilus influenzae to escape host nutritional immunity. Mol. Microbiol. 80, 133-148 (2011).

13. Clantin, B. et al. The crystal structure of filamentous hemagglutinin secretion domain and its implications for the two-partner secretion pathway. Proc. Natl Acad. Sci. USA 101, 6194-6199 (2004).

14. Baelen, S., Dewitte, F., Clantin, B. \& Villeret, V. Structure of the secretion domain of HxuA from Haemophilus influenzae. Acta Crystallogr. Sect. F Struct. Biol. Cryst. Commun. 69, 1322-1327 (2013).

15. Weaver, T. M. et al. Structural and functional studies of truncated hemolysin A from Proteus mirabilis. J. Biol. Chem. 284, 22297-22309 (2009).

16. Yeo, H. J. et al. The structure of the Haemophilus influenzae HMW1 pro-piece reveals a structural domain essential for bacterial two-partner secretion. J. Biol. Chem. 282, 31076-31084 (2007).

17. Buscher, A. Z., Grass, S., Heuser, J., Roth, R. \& St Geme, 3rd J. W. Surface anchoring of a bacterial adhesin secreted by the two-partner secretion pathway. Mol. Microbiol. 61, 470-483 (2006).

18. Morgan, W. T. \& Smith, A. Domain structure of rabbit hemopexin. Isolation and characterization of a heme-binding glycopeptide. J. Biol. Chem. 259, 12001-12006 (1984).

19. De Chiara, M. et al. Genome sequencing of disease and carriage isolates of nontypeable Haemophilus influenzae identifies discrete population structure. Proc. Natl Acad. Sci. USA 111, 5439-5444 (2014).

20. Wandersman, C. \& Delepelaire, P. Haemophore functions revisited. Mol. Microbiol. 85, 618-631 (2012).

21. Deniau, C. et al. Thermodynamics of heme binding to the HasA(SM) hemophore: effect of mutations at three key residues for heme uptake. Biochemistry 42, 10627-10633 (2003).

22. Krieg, S. et al. Heme uptake across the outer membrane as revealed by crystal structures of the receptor-hemophore complex. Proc. Natl Acad. Sci. USA 106, 1045-1050 (2009).

23. Villareal, V. A. et al. Transient weak protein-protein complexes transfer heme across the cell wall of Staphylococcus aureus. J. Am. Chem. Soc. 133, 14176-14179 (2011)

24. Mazar, J. \& Cotter, P. A. New insight into the molecular mechanisms of twopartner secretion. Trends Microbiol. 15, 508-515 (2007).

25. Relman, D. et al. Recognition of a bacterial adhesion by an integrin: macrophage CR3 (alpha M beta 2, CD11b/CD18) binds filamentous hemagglutinin of Bordetella pertussis. Cell 61, 1375-1382 (1990).

26. Grass, S. et al. The Haemophilus influenzae HMW1 adhesin is glycosylated in a process that requires HMW1C and phosphoglucomutase, an enzyme involved in lipooligosaccharide biosynthesis. Mol. Microbiol. 48, 737-751 (2003).

27. St Geme, 3rd J. W. The HMW1 adhesin of nontypeable Haemophilus influenzae recognizes sialylated glycoprotein receptors on cultured human epithelial cells. Infect. Immun. 62, 3881-3889 (1994).

28. Uphoff, T. S. \& Welch, R. A. Nucleotide sequencing of the Proteus mirabilis calcium-independent hemolysin genes (hpmA and hpmB) reveals sequence similarity with the Serratia marcescens hemolysin genes (shlA and shlB). J. Bacteriol. 172, 1206-1216 (1990).

29. Wang, X. et al. Hemolysin EthA in Edwardsiella tarda is essential for fish invasion in vivo and in vitro and regulated by two-component system EsrA-EsrB and nucleoid protein HhaEt. Fish Shellfish Immunol. 29, 1082-1091 (2010).

30. Hertle, R. Serratia type pore forming toxins. Curr. Protein Pept. Sci. 1, 75-89 (2000).

31. Kida, Y., Higashimoto, Y., Inoue, H., Shimizu, T. \& Kuwano, K. A novel secreted protease from Pseudomonas aeruginosa activates NF-kappaB through protease-activated receptors. Cell. Microbiol. 10, 1491-1504 (2008).

32. Clantin, B. et al. Structure of the membrane protein FhaC: a member of the Omp85-TpsB transporter superfamily. Science 317, 957-961 (2007).

33. Hvidberg, V. et al. Identification of the receptor scavenging hemopexin-heme complexes. Blood 106, 2572-2579 (2005).
34. Taketani, S. et al. Hemopexin from four species inhibits the association of heme with cultured hepatoma cells or primary rat hepatocytes exhibiting a small number of species specific hemopexin receptors. Hepatology 27, 808-814 (1998).

35. Cornelissen, C. N. et al. Gonococcal transferrin-binding protein 1 is required for transferrin utilization and is homologous to TonB-dependent outer membrane receptors. J. Bacteriol. 174, 5788-5797 (1992).

36. Wong, J. C., Holland, J., Parsons, T., Smith, A. \& Williams, P. Identification and characterization of an iron-regulated hemopexin receptor in Haemophilus influenzae type b. Infect. Immun. 62, 48-59 (1994).

37. Letoffe, S., Delepelaire, P. \& Wandersman, C. Free and hemophore-bound heme acquisitions through the outer membrane receptor HasR have different requirements for the TonB-ExbB-ExbD complex. J. Bacteriol. 186, 4067-4074 (2004).

38. Letoffe, S. et al. Haemophore-mediated bacterial haem transport: evidence for a common or overlapping site for haem-free and haem-loaded haemophore on its specific outer membrane receptor. Mol. Microbiol. 41, 439-450 (2001).

39. Noinaj, N. et al. Structural basis for iron piracy by pathogenic Neisseria. Nature 483, 53-58 (2012).

40. Dickson, C. F. et al. Structure of the hemoglobin-IsdH complex reveals the molecular basis of iron capture by Staphylococcus aureus. J. Biol. Chem. 289, 6728-6738 (2014).

41. Morgan, W. T. et al. Identification of the histidine residues of hemopexin that coordinate with heme-iron and of a receptor-binding region. J. Biol. Chem. 268, 6256-6262 (1993).

42. Kabsch, W. XDS. Acta Crystallogr. D Biol. Crystallogr. 66, 125-132 (2010).

43. Sheldrick, G. M. A short history of SHELX. Acta Crystallogr. A 64, 112-122 (2008).

44. McCoy, A. J. et al. Phaser crystallographic software. J. Appl. Crystallogr. 40, 658-674 (2007).

45. Perrakis, A., Morris, R. \& Lamzin, V. S. Automated protein model building combined with iterative structure refinement. Nat. Struct. Biol. 6, 458-463 (1999).

46. Emsley, P., Lohkamp, B., Scott, W. G. \& Cowtan, K. Features and development of Coot. Acta Crystallogr. D Biol. Crystallogr. 66, 486-501 (2010).

47. Bricogne, G. et al. BUSTER version 2.11.1. in Cambridge 2.11.1 edn (Global Phasing Ltd., 2011).

48. Chen, V. B. et al. MolProbity: all-atom structure validation for macromolecular crystallography. Acta Crystallogr. D Biol. Crystallogr. 66, 12-21 (2010).

49. Wiseman, T., Williston, S., Brandts, J. F. \& Lin, L. N. Rapid measurement of binding constants and heats of binding using a new titration calorimeter. Anal. Biochem. 179, 131-137 (1989).

50. Tang, G. et al. EMAN2: an extensible image processing suite for electron microscopy. J. Struct. Biol. 157, 38-46 (2007).

51. Letoffe, S., Nato, F., Goldberg, M. E. \& Wandersman, C. Interactions of HasA, a bacterial haemophore, with haemoglobin and with its outer membrane receptor HasR. Mol. Microbiol. 33, 546-555 (1999).

\section{Acknowledgements}

we thank Andrew Thompson from the PROXIMA-1 beamline at SOLEIL for data collection and help for initial model building, Françoise Baleux from the Unité de Chimie des Biomolécules from Institut Pasteur for expert technical assistance in aminoacid analysis and mass spectrometry, Emmanuel Frachon from the Plate-forme Protéines Recombinantes from Institut Pasteur for protein production, and Pascal Arnoux for critical reading. We thank Shirley Mueller for reading the manuscript. B.C. and V.V. are indebted to the Research Federation FRABio (Univ. Lille, CNRS, FR 3688, FRABio, Biochimie Structurale et Fonctionnelle des Assemblages Biomoléculaires) for providing the scientific and technical environment conducive to achieving their contribution. This work was supported by grants from ANR (HEMESTOCKEXCHANGE ANR-12-BSV3-0022-01 and LABEX DYNAMO ANR-11-LABEX-0011-01).

\section{Author contributions}

S.Z., P.D., A.H., M.C. and S.H. performed the experiments. S.Z., P.D., A.H., M.C., S.H., V.V. and B.C. interpreted the results. S.Z., P.D., V.V., A.H. and M.C. wrote the manuscript.

\section{Additional information}

Accession codes: HxuA structure and HxuA-NtHpx structure have been deposited in the PDB under the accession codes 4RM6 (HxuA) and 4RT6 (HxuA-NtHpx).

Supplementary Information accompanies this paper at http://www.nature.com/ naturecommunications

Competing financial interests: The authors declare no competing financial interests. 
Reprints and permission information is available online at http://npg.nature.com/ reprintsandpermissions/

How to cite this article: Zambolin, S. et al. Structural basis for haem piracy from host haemopexin by Haemophilus influenzae. Nat. Commun. 7:11590 doi: $10.1038 /$ ncomms11590 (2016). (c) (i) This work is licensed under a Creative Commons Attribution 4.0 International License. The images or other third party material in this article are included in the article's Creative Commons license, unless indicated otherwise in the credit line; if the material is not included under the Creative Commons license, users will need to obtain permission from the license holder to reproduce the material. To view a copy of this license, visit http://creativecommons.org/licenses/by/4.0/ 